\title{
Critical Behaviour in Baryonic Matter
}

\author{
B. Berg ${ }^{1}$, J. Engels ${ }^{2}$, E. Kehl ${ }^{2}$, B. Waltl ${ }^{2}$, H. Satz ${ }^{3}$ \\ ${ }^{1}$ Department of Physics, Florida State University, Tallahassee, FL 32306, USA \\ ${ }^{2}$ Fakultät für Physik, Universität, D-4800 Bielefeld, Federal Republic of Germany \\ ${ }^{3}$ Fakultät für Physik, Universität, D-4800 Bielefeld, Federal Republic of Germany, and \\ Department of Physics Brookhaven National Laboratory, Upton, NY 11973, USA
}

Received 19 February 1986

\begin{abstract}
First we consider the phenomenology of deconfinement and chiral symmetry restoration for strongly interacting matter at non-vanishing baryon number density. Subsequently, we present numerical results obtained by a Monte Carlo evaluation of statistical QCD on an $8^{3} \times 3$ lattice, using Wilson fermions with $N_{f}=2$, in fourth order hopping parameter expansion, and suppressing the imaginary part of the fermion action. We consider baryonic chemical potentials up to $\mu a=0.6\left(\mu / \Lambda_{L} \simeq 200\right)$; in this range, the critical parameters for deconfinement and chiral symmetry restoration are found to coincide.
\end{abstract}

\section{Introduction}

The prediction of the phase structure of strongly interacting matter is one of the most challenging problems in statistical QCD. We expect that with increasing density, hadronic matter will be transformed into a plasma of coloured, massless quarks and gluons: it should undergo deconfinement and chiral symmetry restoration. The increase in density can be achieved either by heating or by compression, and hence the phase of the system will depend on both temperature and baryon number density.

In the case of vanishing baryon number density, deconfinement and chiral symmetry restoration have been investigated in a variety of lattice evaluation schemes, and the thermodynamics of such "mesonic" matter is slowly emerging [1]. Quantitative studies of the baryon number dependence, however, have been initiated only quite recently $[2,3]$; and this topic will form the main subject of our paper.
To clarify the phenomena which we want to investigate, it seems helpful to first consider a simple schematic model, stripped of all but the essential dynamics. This will be the topic of the first section. Following it, we will turn to lattice QCD at nonvanishing baryonic chemical potential $\mu$. Treating the quarks (we will consider two flavours) as Wilson fermions in low order hopping parameter expansion, we will calculate the basic thermodynamic observables and study the pattern of deconfinement and chiral symmetry restoration in baryonic matter.

\section{Basic Phenomenology}

Let us first look at hadron and quark systems of vanishing baryon number density. Consider an ideal gas of massless pions. Its pressure is

$P_{\pi}=\frac{\pi^{2}}{90} \times 3 \times T^{4}=\frac{\pi^{2}}{30} T^{4}$,

taking into account the three possible charge states. For an ideal plasma of massless quarks, antiquarks and gluons, the pressure becomes

$P_{q}=\frac{\pi^{2}}{90}\left[\frac{7}{8} \times 2 \times 2 \times 2 \times 3+8 \times 2\right] T^{4}=\frac{37 \pi^{2}}{90} T^{4}$,

including two flavours ( $u$ and $d$ ), two spin orientations and three colours for quarks and antiquarks, eight colours and two spin orientations for gluons. Comparing the two states, we note that the pressure of the plasma - with more degrees of freedom always exceeds that of the pion gas. Matter in equilibrium would thus always be in the plasma 


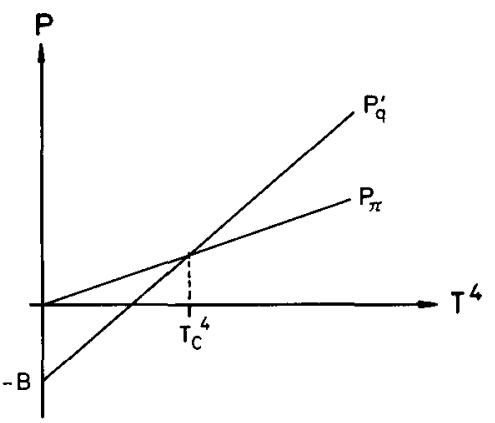

Fig. 1. Ideal pion gas $\left(P_{n}\right)$ vs. ideal quark-gluon plasma with bag pressure $\left(P_{q}^{\prime}\right)$

phase. The essential dynamical input to change this is the (non-perturbative) bag pressure $B$, which reduces $P_{q}$ :

$$
P_{q}^{\prime}=P_{q}-B \text {. }
$$

The result is shown in Fig. 1; there now is a crossover, which determines

$T_{c}=\left(\frac{90}{34 \pi^{2}} B\right)^{1 / 4} \simeq 0.72 B^{1 / 4}$

as transition temperature. - Including the pion mass and/or further resonant states, such as $\rho$ and $\omega$, does not lead to any qualitative change of this picture; neither do perturbative corrections to the quarkgluon plasma.

For baryonic matter, we will now consider the other extreme: $T=0$ at nonzero baryonic chemical potential $\mu$. A perfect Fermi gas of massless protons and neutrons has the pressure

$P_{N}=\frac{1}{24 \pi^{2}} \times 2 \times 2 \times \mu^{4}=\frac{\mu^{4}}{6 \pi^{2}}$.

The ideal quark plasma, with coloured $u$ and $d$ quarks, gives

$P_{q}=\frac{1}{24 \pi^{2}} \times 2 \times 2 \times 3 \times \mu_{q}^{4}=\frac{\mu_{q}^{4}}{2 \pi^{2}}$.

At equilibrium, $\mu_{q}=\mu / 3$, and hence

$P_{q}=\frac{1}{27} \frac{\mu^{4}}{6 \pi^{2}}$.

Here we find that the nuclear matter phase dominates at all $\mu$. To change this, we have to take into account the repulsion between nucleons, which puts a bound on the compression of nuclear matter. For nucleons with hard cores of volume $V_{N}$, the nuclear pressure becomes

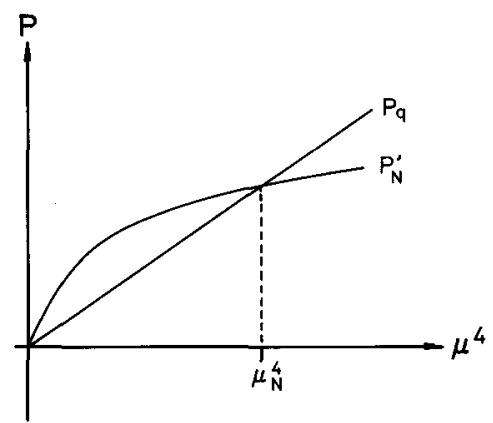

Fig. 2. Ideal Fermi gas of quarks $\left(P_{q}\right)$ vs. ideal Fermi gas of massless nucleons with hard core repulsion $\left(P_{N}^{\prime}\right)$

$P_{N}^{\prime}=\frac{P_{N}}{1+n V_{N}} ;$

here

$n=\frac{2}{3 \pi^{2}} \mu^{3}$

is the density of a perfect Fermi gas of nucleons. Hence we find

$P_{N}^{\prime}=\frac{\mu^{4}}{6 \pi^{2}+4 \mu^{3} V_{N}}$.

The behaviour of $P_{N}^{\prime}$ vs. $P_{q}$ is shown in Fig. 2: the hard core repulsion provides a much weaker growth of $P_{N}^{\prime}$ at large $\mu$ and thus leads to a cross-over, with

$\mu_{c}=\left(\frac{39 \pi^{2}}{V_{N}}\right)^{1 / 3} \simeq 7.27 V_{N}^{-1 / 3}$

as the critical chemical potential. - Here also a more realistic picture, with massive nucleons and bag pressure, does not lead to qualitative changes .

We thus note that on a purely phenomenological level, deconfinement is at $\mu=0$ basically determined by the bag pressure, while at $T=0$, it is the nucleon repulsion which is crucial.

To compare deconfinement and chiral symmetry restoration, we must allow a third phase: constituent quark matter. Consider a non-interacting gas of coloured constituent quarks of mass $m_{Q} \simeq \frac{1}{3} m_{N}$, together with massless pions as Goldstone bosons; relative to the physical vacuum, there will be a confining bag pressure $B_{Q}$, with $\left|B_{Q}\right|<|B|$. For this phase, we have at $\mu=0$

$\star$ The nucleon mass $m_{N}$ does, however, determine a lower bound for the bag pressure, if there is to be a cross-over [4]: from $P_{q}(\mu$ $\left.=m_{N}\right)-B \geqq 0 \quad$ we obtain $B^{1 / 4} \geqq\left(162 \pi^{2}\right)^{-1 / 4} \quad m_{N} \simeq 0.158 m_{N} \simeq 148$ $\mathrm{MeV}$ 


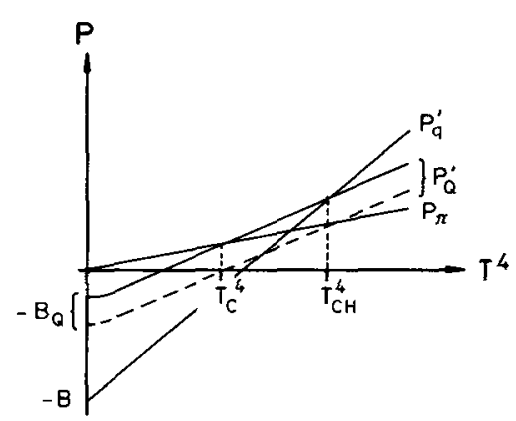

Fig. 3. Constituent quark matter $\left(P_{Q}^{\prime}\right)$, compared to ideal pion gas $\left(P_{n}\right)$ and ideal quark-gluon plasma $\left(P_{q}^{\prime}\right)$

$P_{Q}^{\prime}=\frac{\pi^{2}}{90}\left[21 \frac{P\left(m_{Q}, T\right)}{P(0, T)}+3\right] T^{4}-B_{Q}$

where $P\left(m_{Q}, T\right)$ is the pressure of an ideal gas of massive fermions with one intrinsic degree of freedom. In Fig. 3, we compare (12) to the ideal pion pressure (1) and that of the chirally symmetric plasma of massless quarks. There are now in general two transitions: deconfinement at $T_{c}=f\left(B_{Q}, m_{Q}\right)$ and chiral symmetry restoration at $T_{\mathrm{CH}}=f\left(B_{Q}, B, m_{Q}\right)$. Whether the intermediate constituent quark phase actually occurs, or whether it leads to a pressure below $P_{\pi}$ in the hadronic regime and below $P_{q}^{\prime}$ once the plasma pressure crosses $P_{\pi}$ - the behaviour indicated by the dashed curve in Fig. 3 - depends on the actual values of the parameters involved. Lattice calculations [5-8] have led to $T_{c} \simeq T_{\mathrm{CH}}$ and would thus support the latter case. On a phenomenological level, the question is studied in detail in [9].

At $T=0$, we must add to the properties of constituent quark matter a hard core baryonic repulsion between the quarks, characterized by an intrinsic constituent quark volume $V_{Q}\left(<\frac{1}{3} V_{N}\right)$. The resulting pressure then is

$P_{Q}^{\prime}=\frac{P_{Q}\left(m_{Q}, \mu\right)}{1+n_{Q} V_{Q}}-B_{Q}$

here

$$
\begin{aligned}
P_{Q}\left(m_{Q}, \mu\right)= & \frac{m_{Q}^{4}}{2 \pi^{2}}\left\{\frac{\mu}{3 m_{Q}}\left(\frac{\mu^{2}}{9 m_{Q}^{2}}-1\right)^{1 / 2}\left(\frac{\mu^{2}}{9 m_{Q}^{2}}-\frac{5}{2}\right)\right. \\
& \left.+\frac{3}{2} \ln \left[\frac{\mu}{3 m_{Q}}+\left(\frac{\mu^{2}}{9 m_{Q}^{2}}-1\right)^{1 / 2}\right]\right\}
\end{aligned}
$$

denotes the pressure of an ideal gas of massive fermions [4] with two spin, two flavour and three colour degrees of freedom. Similarly,

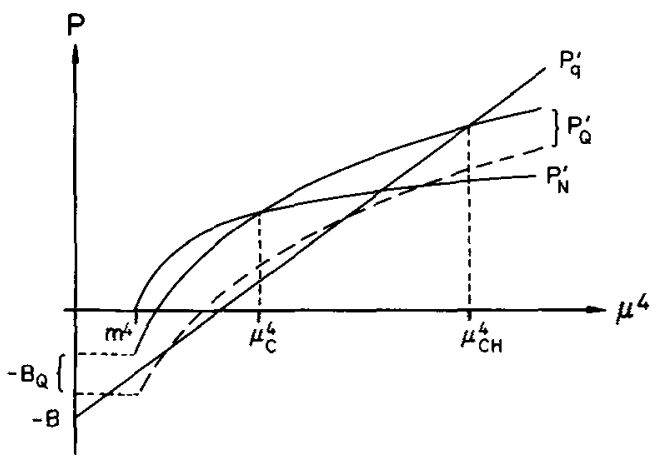

Fig. 4. Constituent quark matter $\left(P_{Q}^{\prime}\right)$, compared to ideal Fermi gas of hardcore nucleons $\left(P_{N}^{\prime}\right)$ and ideal Fermi gas of massless quarks $\left(P_{q}^{\prime}\right)$

$n_{Q}=\frac{2}{27 \pi^{2}}\left(\mu^{2}-9 m_{Q}^{2}\right)^{3 / 2}$

is the baryon number density for the constituent quark system; here, as above, $\mu$ is the baryonic chemical potential. - In Fig. 4, we compare the pressure of constituent quark matter, (13), with that of the plasma of massless quarks and with that of nuclear matter. For the sake of consistency, we have now included the nucleon mass in calculating $P_{N}$, and the bag pressure $B$ in $P_{q}$. Again we obtain in general two transitions: deconfinement at $\mu_{c}$ and chiral symmetry restoration at $\mu_{\mathrm{CH}}$. The crucial question for lattice studies thus is whether these phenomena are indeed distinct, or if they occur at the same value of the baryonic chemical potential.

\section{Lattice QCD at $\mu \neq 0$}

The starting point for statistical QCD is the partition function

$Z(T, \mu)=\operatorname{Tr}\left\{e^{-(H-\mu N) / T}\right\}$,

where $H$ is the Hamiltonian, $\mu$ the baryonic chemical potential, and $N$ the net baryon number of the system. On an asymmetric but isotropic Euclidean lattice with $N_{\sigma}\left(N_{\tau}\right)$ spatial (temporal) lattice sites, the partition function becomes

$Z\left(N_{\sigma}, N_{\tau} ; g^{2} ; \mu\right)=\int \prod_{\text {links }} d U e^{-S_{G}}\{\operatorname{det} Q\}^{N_{f}}$.

Here

$S_{G}(U)=\frac{6}{g^{2}} \sum_{\mathbb{P}}\left(1-\frac{1}{3} \operatorname{Re} \operatorname{Tr} U U U U\right)$

gives the gauge field action as plaquette sum, with $U$ denoting the gauge group elements and $g^{2}$ the bare 
coupling on the lattice. The fermion determinant, $\operatorname{det} Q$, results from the integration of the quark fields, with

$S_{F}=\sum_{f} \bar{\psi}_{f} Q \psi_{f}$

as quark action; we consider here $N_{f}$ massless quark species. In Wilson's formulation [10], $Q$ has the form

$Q=1-\kappa \sum_{v=0}^{3} M_{v} \equiv 1-\kappa M$

with

$\left(M_{v}\right)_{n m}=\left\{\begin{array}{l}\left(1-\gamma_{v}\right) U_{n m} \delta_{n, m-\hat{v}} \\ +\left(1+\gamma_{v}\right) U_{m n}^{+} \delta_{n, m+\hat{v}}, \quad v=1,2,3 \\ \left(1-\gamma_{0}\right) U_{n m} \delta_{n, m-\hat{v}} e^{\mu a} \\ +\left(1+\gamma_{0}\right) U_{m n}^{+} \delta_{n, m+\hat{v}} e^{-\mu a}, \quad v=0 .\end{array}\right.$

Here $U_{n m}$ is the group variable associated to the link between two adjacent sites $n$ and $m ; n+\hat{v}$ denotes the site obtained by a unit shift in the $v$ direction. The strength of the quark interaction is characterized by the hopping parameter $\kappa\left(g^{2}\right) ; a\left(g^{2}\right)$ denotes the lattice spacing. For the sake of simplicity, we shall here write all formulae for equal couplings and equal lattice spacings in space and temperature directions. In actual calculations, they are of course set equal only after all operations (differentiation) are carried out.

The introduction of the chemical potential in (21) follows the prescription of [2] and [11]; a more general form is discussed in [12]. A common feature of all forms is that with $\mu \neq 0$, the $U$ and $U^{+}$terms in (21 b) are no longer hermitean conjugates, and as a consequence, $\operatorname{det} Q$ becomes complex. Note, however, that $Z$ remains real, since both $\int d U$ and $\int d U^{+}$ cover the complete group space.

In the hopping parameter expansion, we obtain for the quark action

$S_{F} \equiv N_{f} \ln \operatorname{det}(1-\kappa M)=-N_{f} \operatorname{Tr} \sum_{l=1}^{\infty} \frac{\kappa^{l}}{l} M^{l}$,

which gives for $N_{\tau} \leqq 4$ as leading terms

$$
\begin{aligned}
S_{F}= & -2 N_{f}(2 \kappa)^{N_{\tau}} \sum_{\text {sites } x}\left\{L_{x} e^{\beta \mu}+L_{x}^{*} e^{-\beta \mu}\right\} \\
& -16 N_{f} \kappa^{4} \sum_{\mathbb{P}} \operatorname{Re} \operatorname{Tr} U U U U+O\left(\kappa^{5}\right),
\end{aligned}
$$

with $\beta \equiv N_{\tau} a$ for the inverse temperature and

$$
L_{x}=\operatorname{Tr} \prod_{\tau=1}^{N_{\tau}} U_{\mathbf{x} ; \tau, \tau+1}
$$

for the thermal Wilson loop at spatial site $\mathbf{x}$; the sum in the first term of (23) runs over all such sites.
It gives in this approximation the main quark contribution, since the second term amounts only to the shift

$6 / g^{2} \rightarrow\left(6 / g^{2}+48 N_{f} \kappa^{4}\right)$

in the gauge field action. Writing the first term of the quark action as

$$
\begin{aligned}
S_{F}(L)= & -4 N_{f}(2 \kappa)^{N_{\tau}} \sum_{x}\left\{\operatorname{Re} L_{x} \cosh \beta \mu\right. \\
& \left.+i \operatorname{Im} L_{x} \sinh \beta \mu\right\}
\end{aligned}
$$

we see explicitly that it is complex for $\mu \neq 0$. From

$$
\begin{aligned}
\operatorname{Im} Z=\int \prod d U e^{-S_{G}-\operatorname{ReS}_{F}} \\
\cdot \cdot \sin \left[-4 N_{f}(2 \kappa)^{N_{\tau}} \sum_{x} \operatorname{Im} L_{x} \sinh \beta \mu\right]
\end{aligned}
$$

we also have explicitly, by changing variables $U \rightarrow U^{+}$, that $\operatorname{Im} Z=0$ and hence $Z$ real. In this order of the hopping parameter expansion we therefore obtain for the partition function

$Z\left(N_{\sigma}, N_{\tau} ; g^{2}, \mu\right)=\int \prod d U e^{-S_{G}^{\prime}-\operatorname{Re} S_{F}(L)} \cos \left[\operatorname{Im} S_{F}(L)\right]$,

where $S_{G}^{\prime}$ denotes the gauge action with the shift (25) and $S_{F}(L)$ is given by (26).

Using the form (28), we now define the thermodynamic average of an quantity $f(U)$ in the usual way

$$
\begin{gathered}
\langle f\rangle \equiv \int \prod d U e^{-S_{G}^{\prime}-\operatorname{Re} S_{F}(L)} f \cos \left(\operatorname{Im} S_{F}(L)\right) / \\
\int \prod d U e^{-S_{G}^{\prime}-\operatorname{Re} S_{F}(L)} \cos \left(\operatorname{Im} S_{F}(L)\right) .
\end{gathered}
$$

From this, we see immediately that

$$
\begin{aligned}
& \left\langle\operatorname{Im} L_{x}\right\rangle=0 \quad \forall \mathbf{x}, \\
& \left\langle\operatorname{Im} S_{F}(L)\right\rangle \sim\left\langle\sum_{x} \operatorname{Im} L_{x}\right\rangle=0,
\end{aligned}
$$

since $\operatorname{Im} L_{x}$ changes sign under the transformation $U \rightarrow U^{+}$. Consider now $\left\langle\operatorname{Im} L_{x}\right\rangle$; the integration over $U$, according to (29), gives us the average over configurations. On the other hand, $\sum_{x} \operatorname{Im} L_{x}$ is the lattice average for a given configuration. For large enough lattices and sufficiently many configurations we expect these two averages to agree, if we are not at a critical point: we can then imagine the large lattice to be obtained by combining sufficiently many equilibrium configurations on a smaller lattice. These arguments have led to the approximation of "partial quenching", in which we set $\operatorname{Im} S_{F}=0$ everywhere [3]. In this case, the usual Monte Carlo evaluation can be carried out with $\exp \left\{-S_{G}^{\prime}-\operatorname{Re} S_{F}(L)\right\}$ as weight, and all results to be presented here are 
obtained in this way. Thus now, with partial quenching,

$$
\langle f\rangle_{R}=\int \prod d U e^{-S_{G}^{\prime}-\operatorname{Re} S_{F}(L)} f / \int \prod d U e^{-S_{G}^{\prime}-\operatorname{Re} S_{F}(L)}
$$

defines the thermodynamic average.

We had initially considered it possible to test this partial quenching by calculating

$$
\langle f\rangle=\left\langle f \cos \left(\operatorname{Im} S_{F}(L)\right)\right\rangle_{R} /\left\langle\cos \left(\operatorname{Im} S_{F}(L)\right)\right\rangle_{R},
$$

i.e., by including $\cos \left(\operatorname{Im} S_{F}(L)\right)$ as part of the observable whose average is to be calculated. It turns out that this procedure is not feasible, for the following reason. On an $8^{3} \times 3$ lattice, we find even after 30,000 lattice sweeps, that $\left\langle\sum_{x} \operatorname{Im} L_{x}\right\rangle$ is of order unity, rather than zero, as required in (31). Hence a Monte Carlo evaluation of (33) without importance sampling to assure $\left\langle\sum_{x} \operatorname{Im} L_{x}\right\rangle=0$ cannot be expected to give reasonable results, since the values of $\cos \left(\operatorname{Im} S_{F}(L)\right)$ obtained with $\exp \left\{-S_{G}^{\prime}-\operatorname{Re} S_{F}(L)\right\}$ as weight for the Metropolis algorithm fluctuate wildly.

If we ignore these difficulties and just calculate e.g. $\langle\operatorname{Re} L\rangle$ according to (33) with $\exp \left\{-S_{G}^{\prime}\right.$ $\left.-\operatorname{Re} S_{F}(L)\right\}$ as Monte Carlo weight, we obtain $\langle\operatorname{Re} L\rangle \simeq\langle\operatorname{Re} L\rangle_{R}$, together with $\left\langle\cos \left(\operatorname{Im} S_{F}(L)\right)\right\rangle \simeq 0$ : a smoothly varying function, such as $\operatorname{Re} L$, becomes uncorrelated from the randomly fluctuating $\cos \left(\operatorname{Im} S_{F}(L)\right)$, and hence can in good approximation be taken outside of the integral. Thus the agreement between $\langle\operatorname{Re} L\rangle$ and $\langle\operatorname{Re} L\rangle_{R}$ is here simply a consequence of the fluctuations of $\cos \left(\operatorname{Im} S_{F}(L)\right)$. In this situation, partial quenching appears to be the most reasonable procedure to follow. - As all averages from here on are defined by (32), we shall now drop the subscript $R$ on the averages \langle\rangle$_{R}$.

To evaluate thermodynamic observables as functions of $\mu$ and $T$, we need explicit expressions for $\kappa\left(g^{2}\right)$ and $a\left(g^{2}\right)$. For the hopping parameter, we shall use the weak coupling form [13]

$\kappa\left(g^{2}\right)=\frac{1}{8}\left[1+0.11 g^{2}+O\left(g^{4}\right)\right]$,

and for the lattice spacing the renormalization group relation with $N_{f}=2$

$a\left(g^{2}\right) \Lambda_{L}=\exp \left\{-\frac{4 \pi^{2}}{29}\left(\frac{6}{g^{2}}\right)+\frac{345}{29^{2}} \log \left[\frac{8 \pi^{2}}{29}\left(\frac{6}{g^{2}}\right)\right]\right\}$.

In both cases we expect some deviations at the $g^{2}$ values actually used; but these relations should suffice to give us at least a reasonable qualitative impression of the resulting critical behaviour.

\section{Numerical Results}

Our evaluation was performed on an $8^{3} \times 3$ lattice with $N_{f}=2$. We have included terms up to $\kappa^{4}$ in the hopping parameter expansion (23). For each $g^{2}$ value, we carried out about 3,000-4,000 lattice sweeps. Using these results, we have studied the $T$ and $\mu$ behaviour of the thermal Wilson loop $\langle\operatorname{Re} L\rangle$ as deconfinement measure, of $\langle\bar{\psi} \psi\rangle$ as chiral symmetry measure, and of the overall energy density $\varepsilon$.

In our study, we have considered that baryonic chemical potential in the range $0 \leqq \mu a \leqq 0.6$, i.e., up to about $\mu \sim 300-400 \mathrm{MeV}$. The reason for stopping here is given by the truncation of the hopping parameter expansion: increasing $\mu$ has a similar effect as increasing $\kappa$ and hence necessitates the inclusion of more terms in (22). To obtain some idea of the error made by including only terms up to order $\kappa^{4}$, we have calculated the energy density of an ideal Fermi gas on an $8^{3} \times 3$ lattice for various $\mu$ values and compared the results with those given by the hopping parameter expansion up to $\kappa^{4}$ for this quantity [14]. The ratio

$\varepsilon_{F}^{\kappa^{4}}\left(8^{3} \times 3\right) / \varepsilon_{F}^{\text {full }}\left(8^{3} \times 3\right)$

varies from 0.96 to 1.09 as $\mu a$ is increased from 0 to 0.6 ; in the $\mu$ range considered, the truncation error thus is $10 \%$ or less for an ideal Fermi gas. For larger $\mu$, the error increases; we have therefore only gone up to $\mu a=0.6$. The full result obtained on an $8^{3} \times 3$ lattice is of course not identical with the ideal gas value in the continuum [15]. However, up to $\mu a$ $=0.6$ the difference between the continuum value and the lattice results with $N_{\tau}=3$ is essentially independent of $\mu$.

Let us note at this point one of the disadvantages of the hopping parameter approach: the truncation error is evidently $N_{\tau}$ dependent, and hence this approach is not very suitable for studying the scaling behaviour of thermodynamic observables.

In Fig. 5, we now show the deconfinement measure $\langle\operatorname{Re} L\rangle$ as function of $6 / \mathrm{g}^{2}$ for different $\mu a$ values. There is a clear shift of the deconfinement point to lower $6 / \mathrm{g}^{2}$, i.e., to lower temperatures, as the baryonic chemical potential increases. At the same time, the change in regimes becomes less abrupt with growing $\mu a$. - In Fig. 6, we show the onset of deconfinement obtained at fixed temperature by increasing $\mu a$. It is seen that a variation of $\mu$ results in a behaviour quite similar to that obtained for a variation in $T$, so that deconfinement can indeed be induced either way.

The behaviour of the overall energy density,

$\varepsilon \equiv\left\{T^{2}(\partial \ln Z / \partial T)_{\mu, V}+\mu T(\partial \ln Z / \partial \mu)_{T, V}\right\} / V$ 


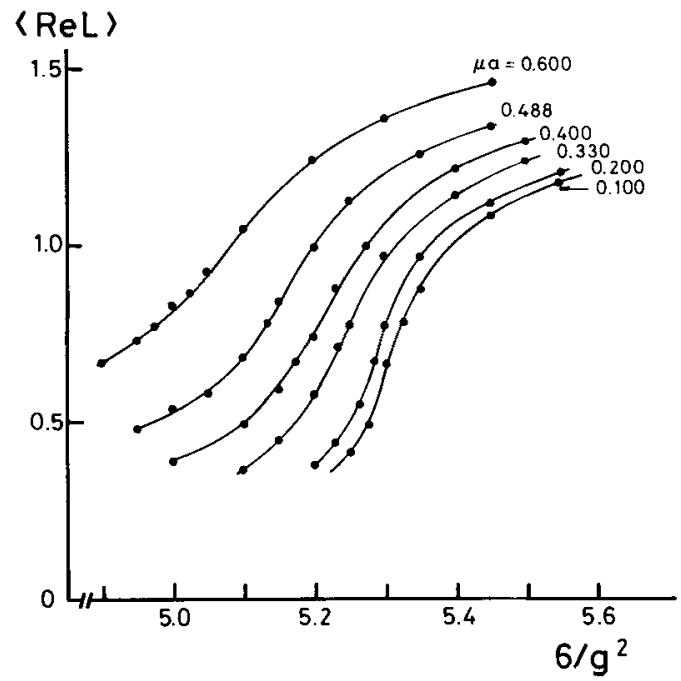

Fig. 5. $\langle\operatorname{Re} L\rangle$ vs. $6 / g^{2}$ for different $\mu a$. Curves are only to guide the eye; naive statistical errors are smaller than the data points

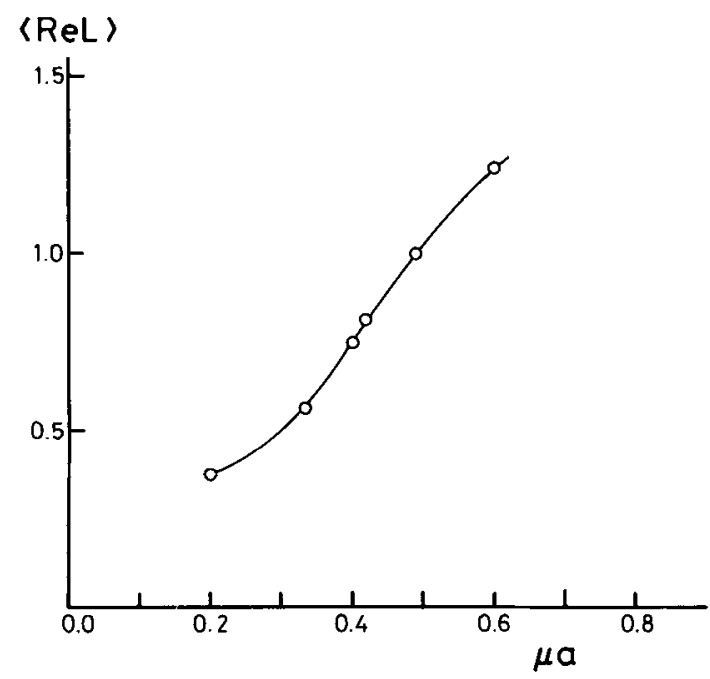

Fig. 6. $\langle\operatorname{Re} L\rangle$ vs. $\mu a$ at $6 / \mathrm{g}^{2}=5.2$; the curve is only to guide the eye

calculated for different $\mu a$ values as function of coupling $6 / \mathrm{g}^{2}$ and temperature $T / \Lambda_{L}$, is shown in Fig. 7 . It is normalized here to the value $\varepsilon_{S B}^{\kappa^{4}}\left(8^{3} \times 3\right)$ for an ideal gas of quarks and gluons, also calculated on an $8^{3} \times 3$ lattice in 4 th order hopping parameter expansion. The lattice evaluation procedure of both $\varepsilon$ and $\varepsilon_{S B}^{k^{4}}\left(8^{3} \times 3\right)$ is described in [16]. - We note in Fig. 7 again a rapid deconfinement transition, becoming slightly "softer" with increasing $\mu a$. In Fig. 8, we show the corresponding behaviour as function of $\mu$ at a fixed value of $T$.

We can now use either the $\varepsilon$ results or those for $\langle\operatorname{Re} L\rangle$ to determine the transition parameters. At $T_{c}, \quad c_{V} \sim(\partial \varepsilon / \partial T)$ should become singular; $\langle\operatorname{Re} L\rangle$ should become exponentially small there. In Fig. 9 ,
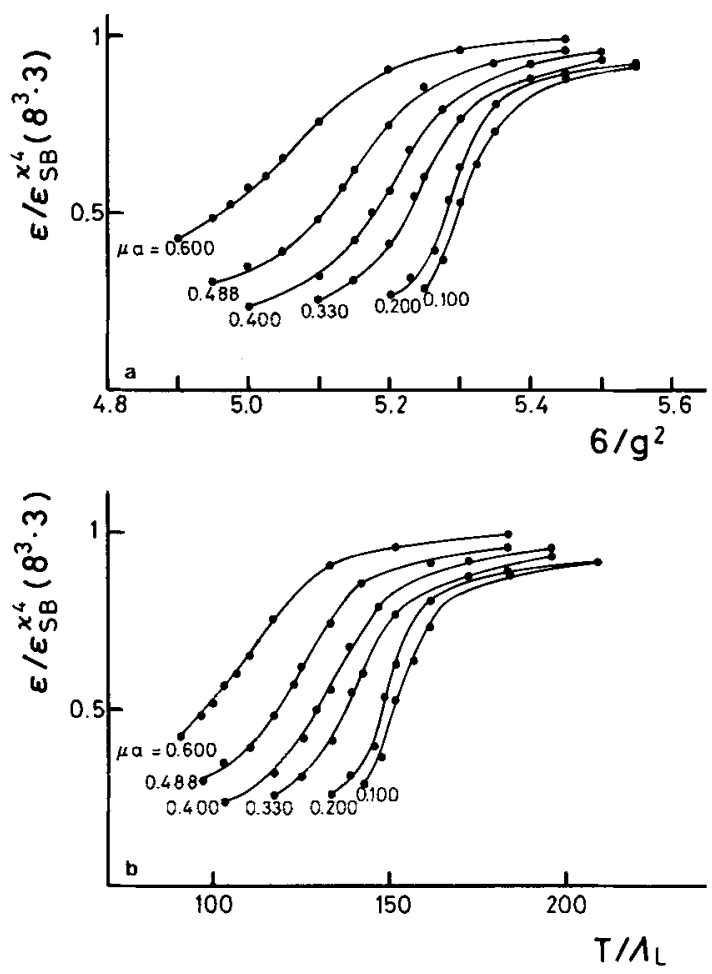

Fig. 7 a, b. Energy density $\varepsilon$, normalized to the corresponding $\kappa^{4}$ ideal gas value on the same lattice, vs. $6 / \mathrm{g}^{2} \mathrm{a}$ and vs. temperature $T / \Lambda_{L} \mathbf{b}$


Fig. 8 a, b. Energy density $\varepsilon$, normalized to the corresponding $\kappa^{4}$ ideal gas value on the same lattice, vs. $\mu a$ a and vs. $\mu / \Lambda_{L} \mathbf{b}$ 


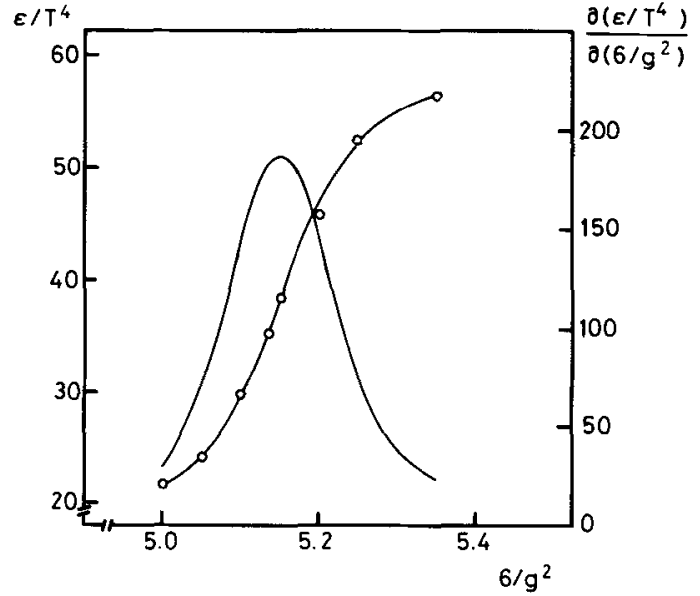

Fig. 9. Energy density and its derivative $(\sim$ specific heat $) \mathrm{vs} .6 / \mathrm{g}^{2}$ at $\mu a=0.488$



Fig. 10. $\langle\operatorname{Re} L\rangle$ and its derivative vs. $6 / \mathrm{g}^{2}$ at $\mu a=0.488$

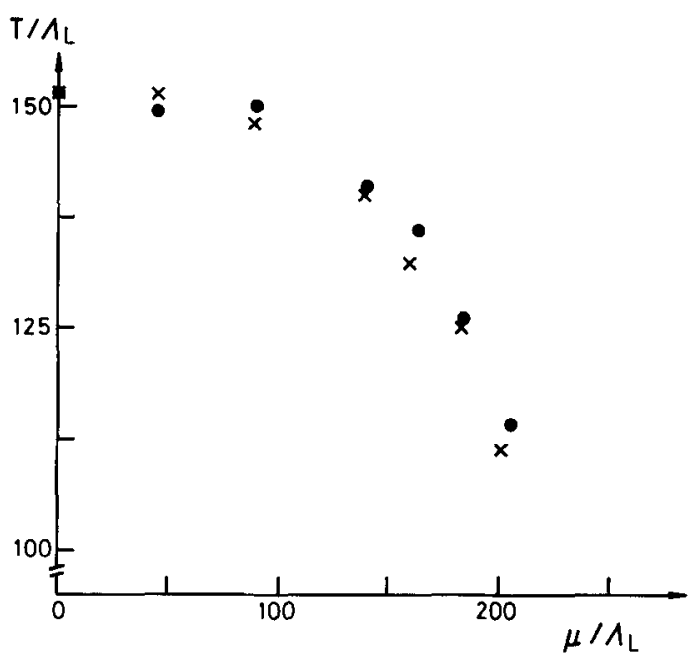

Fig. 11. Phase diagram for deconfinement $(x)$ and chiral symmetry restoration $(\bullet)$

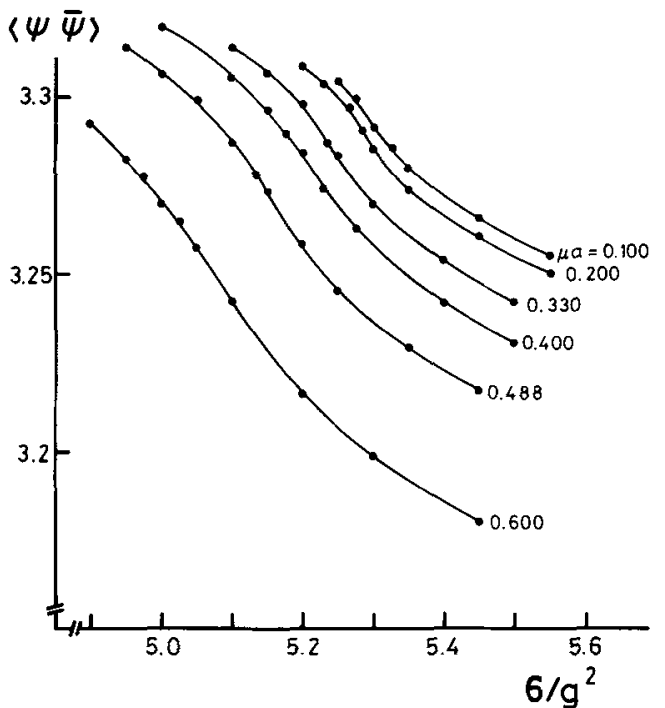

Fig. 12. $\langle\bar{\psi} \psi\rangle$ vs. $6 / g^{2}$ at different $\mu a$

Table 1. Critical parameters for deconfinement and chiral symmetry restoration, as obtained on an $8^{3} \times 3$ lattice

\begin{tabular}{llllllll}
\hline$\mu a$ & $\mu / A_{L}$ & $\begin{array}{l}6 / g_{c}^{2} \\
\text { (from } \varepsilon)\end{array}$ & $\begin{array}{l}T_{d} / A_{L} \\
\text { (from } \varepsilon)\end{array}$ & $\begin{array}{l}6 / g_{c}^{2} \\
\text { (from } \operatorname{Re} L)\end{array}$ & $\begin{array}{l}T_{d} / A_{L} \\
\text { (from } \operatorname{Re} L)\end{array}$ & $\begin{array}{l}6 / g_{c H}^{2} \\
\text { (from } \bar{\psi} \psi \text { ) }\end{array}$ & $\begin{array}{l}T_{\mathrm{CH}} / \Lambda_{2} \\
\text { (from } \bar{\psi} \psi \text { ) }\end{array}$ \\
\hline 0.1 & 45 & 5.299 & 151 & 5.288 & 149 & 5.290 & 150 \\
0.2 & 89 & 5.282 & 148 & 5.294 & 150 & 5.292 & 150 \\
0.33 & 139 & 5.239 & 140 & 5.239 & 140 & 5.243 & 141 \\
0.4 & 161 & 5.194 & 132 & 5.214 & 136 & 5.218 & 136 \\
0.488 & 184 & 5.151 & 125 & 5.158 & 126 & 5.156 & 126 \\
0.6 & 203 & 5.063 & 112 & 5.077 & 114 & 5.081 & 114 \\
\hline
\end{tabular}

we show as an example the behaviour of $\varepsilon / T^{4}$ vs. $6 / g^{2}$ together with its derivative. The critical couplings thus obtained, together with the resulting critical temperatures, are listed in Table 1. Also listed there are the corresponding points of maximum variation of $\langle\operatorname{Re} L\rangle$, and the temperature obtained from them; an illustration of this functional behaviour is shown in Fig. 10. It is seen that the two determinations of $T_{c}(\mu)$ agree quite well, leading to the variation of $T_{c}$ with $\mu$ as shown in Fig. 11. At the highest value of the baryonic chemical potential studied here $\left(\mu / T=1.8\right.$ or $\left.\mu / A_{L} \simeq 200\right)$, the critical 
temperature has dropped by about $25 \%$ :

$T_{c}(\mu=0) / T_{c}\left(\mu / \Lambda_{L} \simeq 200\right) \approx 0.74$.

Finally, we want to consider chiral symmetry restoration and its relation to deconfinement. As is known, the Wilson fermion formulation is not ideal for this purpose, since the chiral symmetry measure $\langle\bar{\psi} \psi\rangle$ never vanishes on a finite lattice. Nevertheless, for $\mu=0$ it is found to show a rapid variation presumably related to the onset of chiral symmetry restoration [16], and it therefore appears meaningful to study the $\mu$-dependence of this variation. The results are shown in Fig. 12; we see again a clear shift to lower turning point values of $6 / \mathrm{g}^{2}$ with increasing $\mu a$. Defining again the critical parameter as that given by the point of maximum variation, we obtain for $\langle\bar{\psi} \psi\rangle$ the values shown in Table 1 . They are seen to agree very well with those obtained for the deconfinement point. We therefore conclude that our results provide up to $\mu a=0.6$ a common point of deconfinement and chiral symmetry restoration. In Fig. 11, we have the resulting phase diagram for both deconfinement and chiral symmetry restoration, as it emerges form the transition parameters listed in Table 1.
Acknowledgement. We thank the Bochum Computer Center and SCRI, Tallahassee, for providing us with facilities (CYBER 205) and computer time.

\section{References}

1. See e.g. H. Satz: Ann. Rev. Nucl. Part. Sci, 35, 245 (1985)

2. J. Kogut et a1.: Nucl. Phys. B225 [FS9], 93 (1983)

3. J. Engels, H. Satz: Phys. Lett. 159 B; 151 (1985)

4. V.V. Dixit, E. Suhonen: Z. Phys. C - Particles and Fields 18 , $355(1983)$

5. J. Polonyi et al.: Phys. Rev. Lett. 53, 644 (1984)

6. R.V. Gavai, M. Lev, B. Petersson: Phys. Lett. 149 B, 492 (1984)

7. F. Fucito, C. Rebbi, S. Solomon: Cal. Tech. Report CALT-68$1127(1984)$

8. T. Çelik, J. Engels, H. Satz: Nucl. Phys. B256, 670 (1985)

9. J. Cleymans, K. Redlich, H. Satz, E. Suhonen: in preparation

10. K. Wilson, in: New phenomena in subnuclear physics. A. Zichichi, ed. New York: Plenum Press 1977

11. P. Hasenfratz, F. Karsch: Phys. Lett. 125B, 308 (1983)

12. R.V. Gavai: Phys. Rev. D32, 519 (1985)

13. N. Kawamoto: Nucl. Phys. B 190 [FS3], 6171 (1981)

14. See e.g. F. Karsch, in: Quark matter formation and heavy ion collisions. M. Jacob, H. Satz, eds. Singapore: World Scientific 1982

15. R.V. Gavai, A. Ostendorf: Phys. Lett. 132B, 137 (1983)

16. T. Çelik, J. Engels, H. Satz: Nucl. Phys. B256, 670 (1985) 